\title{
Toward Personalized Cell Therapies: Autologous Menstrual Blood Cells for Stroke
}

\author{
Maria Carolina O. Rodrigues, ${ }^{1,2,3}$ Loren E. Glover, ${ }^{1}$ Nathan Weinbren, ${ }^{1}$ \\ Jessica A. Rizzi, ${ }^{1}$ Hiroto Ishikawa, ${ }^{1}$ Kazutaka Shinozuka, ${ }^{1}$ Naoki Tajiri, ${ }^{1}$ Yuji Kaneko, ${ }^{1}$ \\ Paul R. Sanberg, ${ }^{1}$ Julie G. Allickson, ${ }^{4}$ Nicole Kuzmin-Nichols, ${ }^{5}$ Svitlana Garbuzova-Davis, ${ }^{1}$ \\ Julio Cesar Voltarelli, ${ }^{2}$ Eduardo Cruz, ${ }^{6}$ and Cesar V. Borlongan ${ }^{1,3}$ \\ ${ }^{1}$ Department of Neurosurgery and Brain Repair, University of South Florida College of Medicine, 12901 Bruce B. Downs Boulevard, \\ Tampa, FL 33612, USA \\ ${ }^{2}$ Department of Internal Medicine, School of Medicine of Ribeirão Preto, University of São Paulo, 14049-900 Ribeirão Preto, \\ SP, Brazil \\ ${ }^{3}$ Department of Neurosurgery and Brain Repair, Center of Excellence for Aging and Brain Repair, University of South Florida College \\ of Medicine, 12901 Bruce B. Downs Boulevard, Tampa, FL 33612, USA \\ ${ }^{4}$ Cryo-Cell International, Inc., Tampa, FL 34677, USA \\ ${ }^{5}$ Saneron-CCEL Therapeutics, Inc. 33612, USA \\ ${ }^{6}$ Cryopraxis, Cell Praxis, BioRio, Póde Biotechnologia do Rio de Janeiro, Rio de Janeiro, Brazil
}

Correspondence should be addressed to Maria Carolina O. Rodrigues, mcarolor@yahoo.com.br and Cesar V. Borlongan, cborlong@health.usf.edu

Received 15 July 2011; Accepted 12 September 2011

Academic Editor: Ken-ichi Isobe

Copyright (C) 2011 Maria Carolina O. Rodrigues et al. This is an open access article distributed under the Creative Commons Attribution License, which permits unrestricted use, distribution, and reproduction in any medium, provided the original work is properly cited.

\begin{abstract}
Cell therapy has been established as an important field of research with considerable progress in the last years. At the same time, the progressive aging of the population has highlighted the importance of discovering therapeutic alternatives for diseases of high incidence and disability, such as stroke. Menstrual blood is a recently discovered source of stem cells with potential relevance for the treatment of stroke. Migration to the infarct site, modulation of the inflammatory reaction, secretion of neurotrophic factors, and possible differentiation warrant these cells as therapeutic tools. We here propose the use of autologous menstrual blood cells in the restorative treatment of the subacute phase of stroke. We highlight the availability, proliferative capacity, pluripotency, and angiogenic features of these cells and explore their mechanistic pathways of repair. Practical aspects of clinical application of menstrual blood cells for stroke will be discussed, from cell harvesting and cryopreservation to administration to the patient.
\end{abstract}

\section{Introduction}

Cell therapy has established itself as an important field of research with considerable progress in the last years. Several disorders, including those of inflammatory, traumatic, degenerative, and autoimmune nature, are listed as potential targets for stem cell application. While the bone marrow leads the investigations, other sources of stem cells have been explored, searching for cells with higher plasticity and tissues with facilitated harvesting [1]. Disposable tissues, such as the amniotic fluid, placenta, and, more recently, menstrual blood, are being investigated as potential sources of stem cells for therapy [2-4]. The immature phenotype, high proliferative potential, and immunomodulatory effects of these cells suggest them as powerful tools for repair.

Neurovascular diseases are the third leading cause of death in the United States and the first cause of chronic disability $[5,6]$. Aging of the population and changes in lifestyle, especially in developed countries, contribute to the progressive increase in the incidence of these disorders, more specifically stroke [7]. On the other hand, treatment is limited, and the only approved therapeutic agent for ischemic stroke is tissue plasminogen activator (tPA). More limiting, however, is the time window for tPA application, restricted to up to 3 
hours after symptom onset [8]. As result, a report from 2008 estimated that only 1.8 to $2.1 \%$ of all stroke patients had been treated with tPA in the United States [9]. It is clear, therefore, that therapeutic alternatives are warranted for the remaining stroke-affected patients which, excluded from tPA benefits, are exposed to the chronic consequences of the disease.

Menstrual blood cell injections are proposed as a restorative therapy after stroke, aiming to provide functional improvement and, therefore, decrease disability of the affected patients. Migration to the site of injury, immunomodulation, and secretion of neurotrophic factors are their main footholds as therapeutic agents. When compared to bone marrow-derived cells, menstrual blood cells present more immature phenotype and behavior, albeit maintaining the characteristic adult stem cell safety [4-10]. Experimental studies have demonstrated benefits of menstrual blood cell administration, with tissue repair and functional improvement, not only in the central nervous system, but also in the heart and ischemic limbs [10-13]. Cell differentiation, although demonstrable in vitro, is still heterogeneously reported in the literature, and its relevance to the final outcome is not yet established. Of more importance may be the endogenous pathways of repair, which are also stimulated by the administrated cells.

This paper aims to characterize menstrual blood cells, describe their possible mechanisms of repair in stroke, and, finally, discuss practical aspects of autologous, thus personalized, application. At this time, however, the use of menstrual blood cells for autologous transplantation is restricted to the female population, regarding autologous use.

\section{The Interaction between Stem Cells and Inflammation in Stroke}

The injury that follows stroke includes the infarct core, which harbors the tissue that evolves almost instantly to death due to ischemia, and the penumbra area, which surrounds the core and that maintains viable, yet nonfunctional, tissue [14]. While the lesion in the core is mostly irreversible, the penumbra area may be rescued, and its fate depends upon severity of the ischemia and time elapsed before restoring blood flow [15]. Treatment with tPA is the first attempt to rescue the penumbra area [16]. Earlier destruction of the clot, restoring blood flow, significantly improves functional outcome of patients. However, application of tPA after the 3-hour time window increases the risk of hemorrhagic transformation of the ischemic stroke, therefore limiting its use to a small, fortunate group of patients that are able to reach the emergency room and complete neurological triage in time to be treated [17]. Therefore, secondary strategies to promote restoration of the penumbra area are strongly warranted.

Inflammation is settled shortly after the ischemic insult and plays a dual role in stroke [18]. Microglial activation leads to the inflammatory events, resulting in blood-brainbarrier leakage, edema, hemorrhage, and leukocyte infiltration $[19,20]$. The migration of attracted cells from the systemic circulation amplifies the inflammatory response, that culminates with release of cytokines, nitric oxide and free radicals, further microglial and astrocytic activation, all of which contribute to the worsening of the neurotoxic environment. Therefore, while inflammation is important to promote clearance of debris, scavenge excess neurotransmitters from the extracellular space, control water and ion homeostasis, and increase the production of neurotrophic factors, it may also promote secondary damage to the nervous tissue [21]. Additionally, the scar tissue generated by the reactive astrocytes, encapsulating the infarcted area and protecting the remaining healthy brain from further damage, interferes with the repairing mechanisms, such as migration of stem cells and angiogenesis. In summary, inflammation is necessary and beneficial in the initial hours after stroke, however, as time progresses, interventions are needed to restrain the aberrant immunological response and thus protect the brain from further damage.

Cell therapy modulates the inflammatory response at the same time that stimulates repairing pathways. The results are improved when the cells are applied according to the dynamics of the inflammatory response. Therefore, interventions in the first 24 hours following stroke are mainly neuroprotective [22] and aim to restore vessel permeability. Treatment with tPA is indicated in this phase, therefore preventing further neuronal death. Thereafter, inflammation settles itself, with maximum intensity during the first week after stroke, during which the stem cells have their best applications, promoting restoration of the penumbra area. Injected intravenously, stem cells migrate to the site of injury and opportunely interact with the inflammatory environment, modulating its deleterious effects and at the same time maximizing its beneficial aspects [23]. Changes in the immunological profile towards a less inflammatory response, increasing of cells with regulatory function, decreasing of inflammatory cytokines and reversal of astrocytic reactivity are described as modulatory effects of stem cells [24,25]. Moreover, cytokines and chemokines secreted by the damaged tissue can stimulate the differentiation of the injected cells into cell types of interest for repair [26]. Still in the inflammatory context, stem cells secrete neurotrophic factors, which in turn stimulate neuronal survival and endogenous repairing pathways $[23,25]$. Once inflammation decreases, after approximately one month after stroke, according to animal studies, cells are no longer attracted to the damage site or are stimulated to differentiate in situ [27]. However, repair can still be promoted through local implantation of predifferentiated cells.

The use of undifferentiated cells through intravenous route seems to be the best approach for clinical translation since it is minimally invasive and possesses multiple therapeutic pathways. On one end of the spectrum of differentiation, embryonic cells have the advantage of high plasticity, trophic support, and proliferation $[28,29]$. However, the risks of uncontrolled proliferation and teratogenicity, among other problems associated with this cell type, make them prohibitive as therapeutic agents. On the other end are the adult stem cells, which still maintain a certain variability in the scale of maturity, according to their source. Therefore, bone marrow-derived stem cells present lower proliferative capacity and less telomerase activity than stem cells derived from adipose, dental pulp, and endometrial tissue. These, 
in turn, present more immature characteristics, expressing embryonic markers and differentiating in vitro into cell types from all three germ cell layers $[1,30]$.

\section{Characterization of Endometrial-Derived Cells}

The presence of stem cells in the endometrium was first described over 30 years ago [31]. The monthly shedding of the superficial layers suggested that cells with high proliferating capacities were present in the tissue. The endometrium is composed of epithelial cells, identified in the superficial layers of the tissue, and extending toward the interface with the myometrium, through the tubular glands. The remaining endometrium consists of stromal cells, smooth muscle cells, endothelial cells, and leukocytes [32]. Functionally, the endometrium can be divided in an upper layer, named functionalis, which contains mostly glands loosely held together by stromal tissue, and in a lower layer, basalis, consisting of dense stroma and branching glands. The functionalis is eliminated monthly, as menstruation, and the basalis persists and gives rise to the new endometrium, under hormonal influence. Against the initial belief that the stem cells were exclusively part of the basalis layer of the endometrium, and were not eliminated with menstruation, Meng et al. [33] detected stem cells in the menstrual blood. Several other studies followed, confirming the discovery $[4,34,35]$ and consolidating menstrual blood as a possible source of stem cells.

Epithelial and stromal cells isolated from the endometrium or from menstrual blood and cultured in vitro show clonogenicity and proliferative capacity, but the epithelial cells soon lose part of their phenotypic markers and need a feeder layer to survive [33, 36]. Patel et al. [4] published a detailed study, in which stromal stem cells isolated from menstrual blood (MenSCs) were expanded in vitro, and showed clonogenic properties and multipotentiality. They also demonstrated that MenSCs expressed markers of pluripotency, such as Oct-4, SSEA-4, and c-kit, which are frequently found in more immature cell types, including the embryonic stem cells.

Recently, Allickson et al. [34] published a study about the proliferative properties of human menstrual blood-derived cells. Agreeing with previous studies, the cells presented high proliferative rates and immature phenotype, expressing embryonic cell markers, which remained unaltered after 20 culture passages. Interestingly, the cells demonstrated resistance, since they were able to be processed up to 96 hours after collection, high viability after processing, and longevity, as some of the cultures were able to be subcultured 47 times before senescence.

\section{Experimental and Clinical Applications of Endometrial-Derived Stem Cells}

Cui et al. published in 2007 the first report of an in vivo application of endometrial-derived cells. In this study, the investigators evaluated the effects of both endometrial cells and menstrual blood cells in a murine model of Duchenne muscular dystrophy. Fusion of the injected cells to myoblasts was observed in vivo and in vitro, followed by the production of human dystrophin by the treated muscle [35]. Shortly after that, Hida et al. [10] described their experience in differentiating menstrual blood-derived stromal cells in vitro into spontaneously beating cardiomyocyte-like cells. When menstrual blood cells were injected in the ischemic tissue of myocardial infarct rat models, functional improvement was noted, differently than what was observed when bone marrow stromal cells were used. Additionally, they compared the phenotype and proliferative characteristics of endometrial gland mesenchymal cells and menstrual blood mesenchymal cells, concluding that they are very similar populations of cells, the latter possibly deriving from the former. Finally, the authors also described cell engraftment and transdifferentiation into cardiac tissue, which seems to be a unique characteristic of this transplantation site, since other studies transplanting endometrial-derived cells into different tissues failed to detect expressive differentiation [11].

Regarding neurovascular disorders, Borlongan et al. [11] reported the results of menstrual blood cell transplantation in experimental stroke. Stromal-like menstrual blood stem cells were isolated, expanded, and selected for CD117, a marker associated with high proliferation, migration, and survival [37]. In vitro studies showed that the expanded cells maintained expression of embryonic-like stem cell phenotypic markers, such as Oct4, SSEA-4, and Nanog, even when cultured up to 9 passages, as an evidence of the safety and reliability of these cells, and some were induced to express neural markers (MAP2 and Nestin). Moreover, when added to cultured rat neurons exposed to a hypoxic insult, the menstrual blood cells provided neuroprotection, and when applied to rat stroke models, less neurologic deficit was observed on functional tests, irrespective of the injection site, that is, systemic or local administration into the striatum. However, analysis of the tissue, after animal sacrifice, revealed that although human cells were detected in the rat brain, some migrating to areas other than the injected, they did not show signs of differentiation, expressing their original markers. The observation suggests that, at least in the brain tissue, cell differentiation is not the main mechanism of repair.

Wolff et al. [13] reported the use of endometrial-derived cells in a Parkinson's disease mouse model. Endometrialderived stromal cells were differentiated in vitro into dopaminergic-like cells, which expressed nestin and tyrosine hydroxylase $(\mathrm{TH})$, an enzyme that participates in dopamine synthesis. Labeled human endometrium-derived cells and predifferentiated dopaminergic-like cells were transplanted into the striatum of the animals. Endometrial-derived stromal cells were able to migrate to the substantia nigra and also showed signs of in vivo differentiation, acquiring neuronal phenotype and expressing human TH. Taken together, these observations demonstrate the therapeutic potential of these cells to functionally restore the damaged tissue. They also reinforce the idea that cells do not need to be predifferentiated before transplantation and that more immature, less-differentiated cell types migrate easily to the inflammatory sites. In contrast to the observations of 
Borlongan et al. [11], widespread cell differentiation was suggested by this study.

The only clinical study yet published evaluated the safety aspects of endometrial-derived stromal cells administration [38]. Four patients with multiple sclerosis were treated with intrathecal injections of 16 to 30 million cells, and one of the patients also received an additional intravenous injection. No adverse events were registered and, in the short followup of 12 months, the authors reported functional stabilization.

Endometrium-derived cells present a strong angiogenic potential that contributes to the experimental investigations of vascular growth and remodeling and, perhaps, even for designing clinical therapeutic studies, as these cells might be applied to cardiovascular diseases. The angiogenic behavior was already predicted by the observation of high levels of VEGF and its receptors in the tissue and is probably associated with the function of the cells in the endometrium, that is, rapid proliferation and implantation of the embryo [39]. In a pilot experimental study, Murphy et al. [12] demonstrated that intramuscular injections of endometrialderived cells in hind limbs of rats were able to prevent the formation of necrotic ulcers after ligation of the femoral artery and its branches. The authors and others propose to investigate the angiogenic properties of these cells in chronic limb ischemia patients and, more recently, severe skin burns, using the cells associated to intelligent artificial films [40].

In summary, the available evidence regarding menstrual blood-derived cells favors their future application in clinical studies. In comparison to stem cells from other sources, especially those from the bone marrow, menstrual bloodderived stem cells have the advantage of presenting a more immature phenotype, through the expression of embryoniclike surface markers. Their immature behavior is confirmed by in vitro differentiation studies in which menstrual bloodderived cells originate diverse tissue types from all three germ layers $[4,33]$. Moreover, they seem to have a higher proliferative capacity, above 30 population doublings, when compared to stromal cells from other sources, such as the bone marrow and dental pulp, which are limited to approximately 20 population doublings [1]. Additionally, cultured menstrual blood cells maintain longer telomerase activity than bone marrow-derived cells $[4,34]$, indicating delayed senescence. These observations may reflect higher regenerative and differentiation potentials in vivo, yet to be confirmed by comparative studies between cells from different sources. Whether endometrial-derived stem cells provide repair through cell differentiation or through paracrine effects, stimulating endogenous repairing pathways, is a point still to be established. The available evidence suggests that the type of tissue and possibly the nature of the injury may determine the repairing mechanisms.

\section{Personalized Therapy: Practical Aspects}

Personalized medicine is a rapidly expanding field offering patient-specific therapies to treat disease. Autologous cell transplantation may be a form of personalized medicine that could afford many benefits in the clinical setting, such as the elimination of graft versus host disease. Transplantation of autologous stem cells may circumvent the need for immunosuppressants, which can cause many deleterious side effects to the patient. Increased availability is another advantage of autologous stem cell transplantation. Ethical controversy surrounding the collection of a patient's own stem cells is greatly reduced, thus increasing the accessibility of these cells. Unlike embryonic and fetal stem cells, harvesting autologous stem cells from the patient does not harm another organism. Embryonic stem cells carry an increased risk of tumor formation, a characteristic not exhibited in autologous adult stem cells. Increased safety and decreased ethical controversy make autologous stem cells an appealing therapeutic option for neurological disease. The debate currently surrounding not only embryonic stem cell retrieval but also stem cell usage in general has sparked issues with the usage. Obtaining cells from a patient's own body would circumvent the ethical controversy as it is not harvesting viable cells from one individual donor to another individual recipient, but it is entirely for the same patient. Additionally, these cells would not be taken in ways possibly deemed as "therapeutic cell cloning," such as the current debate following embryonic and fetal stem cells.

Menstrual blood cells are a promising source of repair. Their immature behavior warrants migration, immunomodulation, secretion of growth factors, and, in some cases, differentiation. These properties, coupled to the angiogenic potential of the cells, make them attractive for restorative approaches following ischemic stroke, as already demonstrated by translational research [11]. Moreover, these cells are optimal candidates for autologous therapy, following the current trend to cryopreserve biological products intended for future use. Although stromal cells have low immunogenicity due to the lack of MHC class II expression [41], therefore enabling allogeneic application, autologous use is still preferred. Some advantages are guaranteed lack of immunogenicity, reverting in longer cell survival and no induction of local inflammatory reaction, safety, and diminished risk of ethical conflicts.

The one-week time window that follows stroke seems the best opportunity for cell therapy. While inflammation is at its best degree, there is enough time to stabilize the patient, complete physical examination and laboratory tests, discuss the therapeutic options with the patient and family, and, finally, apply the cells. Unfortunately, the time window is still short for expansion of autologous cells in vitro, as the procedure usually requires a few weeks to obtain minimum number of cells. Menstrual blood cell banking, thus, seems to be the best strategy and may become a strong competitor to the already available umbilical cord blood banks.

Safety is one important concern regarding the use of menstrual blood. Since the blood is collected through the placement of a silicone cup inside the donor's vagina, therefore exposed to microbial contamination, some precautions are required, such as refrigeration of the collected specimens and use of antibiotics [34]. Additionally, the cells should be processed, expanded, and stored in a certified facility, under strict rules of cleanliness and safety. Additional safety measures include phenotypical verification of the cells after expansion, ensuring that they maintain the original markers 


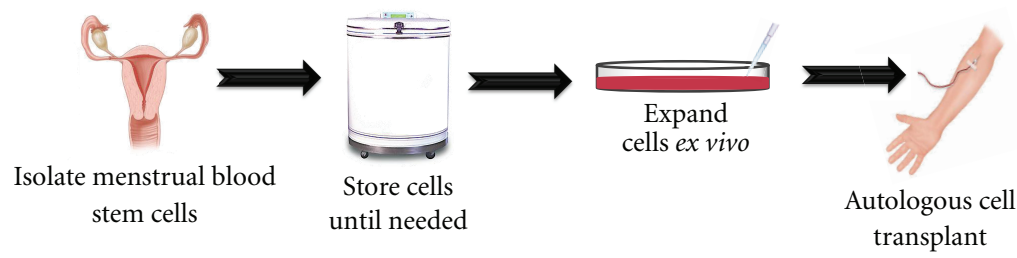

FIGURE 1: Isolation and transplantation of autologous menstrual blood-derived cells. Menstrual blood cells can be collected and stored prior to injury or the development of a neurodegenerative disease. At the time of injury, the cells can then be thawed and expanded ex vivo. Once the ideal number of cells has been reached, the autologous menstrual blood-derived cells can be transplanted into the patient.

and cytogenetic evaluations, excluding chromosomal aberrations and microbiological tests.

A hypothetical scenario would be a woman, in postmenopausal age, recently affected by an ischemic stroke. Due to the limited timeframe between the beginning of symptoms and final diagnosis, she would be part of the majority of patients that are excluded from tPA treatment. After further examinations, evaluation of the extension of the infarct and prognostic evaluations would be completed, estimating the degree of future, long-term disability. Finally, given the information that the patient has menstrual blood cells cryopreserved and ready for use, the therapy would be considered. After patient or familial consent, the cell banking facility would be contacted by the responsible physician, settling date and time for the infusion. The cells would be transported cryopreserved, being thawed at bedside and immediately infused intravenously, under physician supervision and cardiorespiratory monitoring. A second peripheral venous line would be available for emergency medication, if needed. After infusion, the patient would stay in the hospital during at least 24 hours, for adverse reaction monitoring, especially due to the possible toxic effects of dimethyl sulfoxide (DMSO) used in cell cryopreservation and to remaining traces of antibiotics in the cell suspension (Figure 1). After discharge, periodical evaluations would evaluate the patient's progress, establishing goals for the complimentary treatments such as physical and speech therapies, nutrition, and, finally, ability to resume work.

For a stroke-affected patient, the slightest improvement in neurological function can be decisive for self-sufficiency and, even, for ability to work, which are ultimately translated into financial independence. Therefore, investing in cell banking as a safety measure against possible future events may be a wise and even profitable step. While cell banking is already widely accessible for umbilical cord blood, only recently has it also become available for menstrual blood cells. Women in child-bearing age may donate multiple samples of menstrual blood, enabling storage of large amounts of cells for future use. As a further possibility, the cells could be expanded and differentiated into specific tissues and be ready for eventual transplantation use [42]. An efficient banking system for menstrual blood cells would require an organized and updated registration system, enabling prompt identification and rapid retrieval of the cryopreserved cells, just in time for therapeutic application.

\section{Conclusions}

The rescue of the penumbra area after stroke is decisive for functional outcome and a great opportunity for cell therapy [16]. Stem cells promote functional restoration especially through modulation of the activated immune system and secretion of trophic factors [25]. Although cell differentiation is observed in the experimental setting, its importance to the final outcome of the treatment is still undefined. Menstrual cells combine characteristics that are convenient for clinical application and, in parallel with cells derived from other disposable tissues, may have a role in the future investigations. Cryopreservation of autologous cells for future use may be a prudent strategy to those patients at risk of being affected by stroke. Obviously, menstrual blood as a source of autologous cells is limited to women as the target patient population. Despite the potential challenges still to be resolved, these cells represent important therapeutic tools that may improve the disease outcome, decreasing the mortality and morbidity of stroke patients.

\section{Disclosure}

CVB and PRS serve as consultants, and PRS is a cofounder of Saneron-CCEL Therapeutics, Inc., and CVB, PRS, and JGA have a patent application in this area, owned jointly by Cryo-Cell International, Inc. and Saneron-CCEL Therapeutics, Inc. Cryo-Cell International, Inc. provided the foundational menstrual stem cell technology in the patent applications of M. A. Walton and JGA wholly owned by Cryo-Cell International, Inc.

\section{References}

[1] C. E. Gargett, K. E. Schwab, R. M. Zillwood, H. P. T. Nguyen, and $\mathrm{D}$. $\mathrm{Wu}$, "Isolation and culture of epithelial progenitors and mesenchymal stem cells from human endometrium," Biology of Reproduction, vol. 80, no. 6, pp. 1136-1145, 2009.

[2] I. Antonucci, L. Stuppia, Y. Kaneko et al., "Amniotic fluid as a rich source of mesenchymal stromal cells for transplantation therapy," Cell Transplantation, vol. 20, no. 6, pp. 789-795, 2011.

[3] A. Kranz, D. C. Wagner, M. Kamprad et al., "Transplantation of placenta-derived mesenchymal stromal cells upon experimental stroke in rats," Brain Research, vol. 1315, pp. 128-136, 2010. 
[4] A. N. Patel, E. Park, M. Kuzman, F. Benetti, F. J. Silva, and J. G. Allickson, "Multipotent menstrual blood stromal stem cells: isolation, characterization, and differentiation," Cell Transplantation, vol. 17, no. 3, pp. 303-311, 2008.

[5] J. Xu, D. Kenneth, M. A. Kochanek, L. Sherry, and B. S. Murphy, "Deaths: final data for 2007," National Vital Statistics Reports, vol. 57, pp. 1-134, 2010.

[6] Centers for Disease Control and Prevention (CDC), "Prevalence of disabilities and associated health conditions among adults_-United states, 1999," Morbidity and Mortality Weekly Report, vol. 50, pp. 120-125, 2001.

[7] B. Ovbiagele and M. N. Nguyen-Huynh, "Stroke epidemiology: advancing our understanding of disease mechanism and therapy," Neurotherapeutics, vol. 8, no. 3, pp. 319-329, 2011.

[8] J. R. Marler, "Tissue plasminogen activator for acute ischemic stroke," The New England Journal of Medicine, vol. 333, no. 24, pp. 1581-1587, 1995.

[9] D. Kleindorfer, C. J. Lindsell, L. Brass, W. Koroshetz, and J. P. Broderick, "National US estimates of recombinant tissue plasminogen activator use: ICD-9 codes substantially underestimate," Stroke, vol. 39, no. 3, pp. 924-928, 2008.

[10] N. Hida, N. Nishiyama, S. Miyoshi et al., "Novel cardiac precursor-like cells from human menstrual blood-derived mesenchymal cells," Stem Cells, vol. 26, no. 7, pp. 1695-1704, 2008.

[11] C. V. Borlongan, Y. Kaneko, M. Maki et al., "Menstrual blood cells display stem cell-like phenotypic markers and exert neuroprotection following transplantation in experimental stroke," Stem Cells and Development, vol. 19, no. 4, pp. 439451, 2010.

[12] M. P. Murphy, H. Wang, A. N. Patel et al., "Allogeneic endometrial regenerative cells: an "Off the shelf solution" for critical limb ischemia?" Journal of Translational Medicine, vol. 6, article 45, 2008.

[13] E. F. Wolff, X. B. Gao, K. V. Yao et al., "Endometrial stem cell transplantation restores dopamine production in a Parkinson's disease model," Journal of Cellular and Molecular Medicine, vol. 15, no. 4, pp. 747-755, 2011.

[14] M. Fisher, "The ischemic penumbra: a new opportunity for neuroprotection," Cerebrovascular Diseases, vol. 21, supplement 2, pp. 64-70, 2006.

[15] A. R. Green, T. Odergren, and T. Ashwood, "Animal models of stroke: do they have value for discovering neuroprotective agents?" Trends in Pharmacological Sciences, vol. 24, no. 8, pp. 402-408, 2003.

[16] J. C. Chavez, O. Hurko, F. C. Barone, and G. Z. Feuerstein, "Pharmacologic interventions for stroke: looking beyond the thrombolysis time window into the penumbra with biomarkers, not a stopwatch," Stroke, vol. 40, no. 10, pp. e558e563, 2009.

[17] W. Hacke, M. Kaste, E. Bluhmki et al., "Thrombolysis with alteplase 3 to 4.5 hours after acute ischemic stroke," The New England Journal of Medicine, vol. 359, no. 13, pp. 1317-1329, 2008.

[18] J. Kriz, "Inflammation in ischemic brain injury: timing is important," Critical Reviews in Neurobiology, vol. 18, no. 1-2, pp. 145-157, 2006.

[19] S. Amor, F. Puentes, D. Baker, and P. Van Der Valk, "Inflammation in neurodegenerative diseases," Immunology, vol. 129, no. 2, pp. 154-169, 2010.

[20] H. C. A. Emsley, C. J. Smith, P. J. Tyrrell, and S. J. Hopkins, "Inflammation in acute ischemic stroke and its relevance to stroke critical care," Neurocritical Care, vol. 9, no. 1, pp. 125$138,2008$.
[21] T. Takano, N. A. Oberheim, M. L. Cotrina, and M. Nedergaard, "Astrocytes and ischemic injury," Stroke, vol. 40, no. 3, supplement, pp. S8-S12, 2009.

[22] D. C. Hess and C. V. Borlongan, "Cell-based therapy in ischemic stroke," Expert Review of Neurotherapeutics, vol. 8, no. 8, pp. 1193-1201, 2008.

[23] D. H. Park, D. J. Eve, J. Musso et al., "Inflammation and stem cell migration to the injured brain in higher organisms," Stem Cells and Development, vol. 18, no. 5, pp. 693-701, 2009.

[24] L. Bai, D. P. Lennon, V. Eaton et al., "Human bone marrowderived mesenchymal stem cells induce Th2-polarized immune response and promote endogenous repair in animal models of multiple sclerosis," Glia, vol. 57, no. 11, pp. 1192-1203, 2009.

[25] C. Boucherie and E. Hermans, "Adult stem cell therapies for neurological disorders: benefits beyond neuronal replacement?" Journal of Neuroscience Research, vol. 87, no. 7, pp. 1509-1521, 2009.

[26] Y. Fujiwara, N. Tanaka, O. Ishida et al., "Intravenously injected neural progenitor cells of transgenic rats can migrate to the injured spinal cord and differentiate into neurons, astrocytes and oligodendrocytes," Neuroscience Letters, vol. 366, no. 3, pp. 287-291, 2004.

[27] W. D. Hill, D. C. Hess, A. Martin-Studdard et al., "SDF-1 (CXCL12) is upregulated in the ischemic penumbra following stroke: association with bone marrow cell homing to injury," Journal of Neuropathology and Experimental Neurology, vol. 63, no. 1, pp. 84-96, 2004.

[28] A. S. Daar, A. Bhatt, E. Court, and P. A. Singer, "Stem cell research and transplantation: science leading ethics," Transplantation Proceedings, vol. 36, no. 8, pp. 2504-2506, 2004.

[29] Y. Takagi, M. Nishimura, A. Morizane et al., "Survival and differentiation of neural progenitor cells derived from embryonic stem cells and transplanted into ischemic brain," Journal of Neurosurgery, vol. 103, no. 2, pp. 304-310, 2005.

[30] Y. Ikegame, K. Yamashita, S. I. Hayashi et al., "Comparison of mesenchymal stem cells from adipose tissue and bone marrow for ischemic stroke therapy," Cytotherapy, vol. 13, no. 6, pp. 675-685, 2011.

[31] V. A. Prianishnikov, "On the concept of stem cell and a model of functional-morphological structure of the endometrium," Contraception, vol. 18, no. 3, pp. 213-223, 1978.

[32] H. A. Padykula, "Regeneration in the primate uterus: the role of stem cells," Annals of the New York Academy of Sciences, vol. 622, pp. 47-56, 1991.

[33] X. Meng, T. E. Ichim, J. Zhong et al., "Endometrial regenerative cells: a novel stem cell population," Journal of Translational Medicine, vol. 5, article 57, 2007.

[34] J. G. Allickson, A. Sanchez, N. Yefimenko, C. V. Borlongan, and P. R. Sanberg, "Recent studies assessing the proliferative capability of a novel adult stem cell identified in menstrual blood," Open Stem Cell Journal, vol. 3, pp. 4-10, 2011.

[35] C. H. Cui, T. Uyama, K. Miyado et al., "Menstrual bloodderived cells confer human dystrophin expression in the murine model of duchenne muscular dystrophy via cell fusion and myogenic transdifferentiation," Molecular Biology of the Cell, vol. 18, no. 5, pp. 1586-1594, 2007.

[36] R. W. S. Chan, K. E. Schwab, and C. E. Gargett, "Clonogenicity of human endometrial epithelial and stromal cells," Biology of Reproduction, vol. 70, no. 6, pp. 1738-1750, 2004.

[37] N. H. Cho, Y. K. Park, Y. T. Kim, H. Yang, and S. K. Kim, "Lifetime expression of stem cell markers in the uterine 
endometrium," Fertility and Sterility, vol. 81, no. 2, pp. 403407, 2004.

[38] Z. Zhong, A. N. Patel, T. E. Ichim et al., "Feasibility investigation of allogeneic endometrial regenerative cells," Journal of Translational Medicine, vol. 7, article 15, 2009.

[39] X. Fan, S. Krieg, C. J. Kuo et al., "VEGF blockade inhibits angiogenesis and reepithelialization of endometrium," FASEB Journal, vol. 22, no. 10, pp. 3571-3580, 2008.

[40] H. Drago, G. H. Marín, F. Sturla et al., "The next generation of burns treatment, intelligent films and matrix, controlled enzymatic debridement, and adult stem cells," Transplantation Proceedings, vol. 42, no. 1, pp. 345-349, 2010.

[41] A. Uccelli, L. Moretta, and V. Pistoia, "Immunoregulatory function of mesenchymal stem cells," European Journal of Immunology, vol. 36, no. 10, pp. 2566-2573, 2006.

[42] M. J. Zhang, B. Liu, W. Xia, Z. Y. Sun, and K. H. Lu, "Could cells from menstrual blood be a new source for cell-based therapies?" Medical Hypotheses, vol. 72, no. 3, pp. 252-254, 2009. 

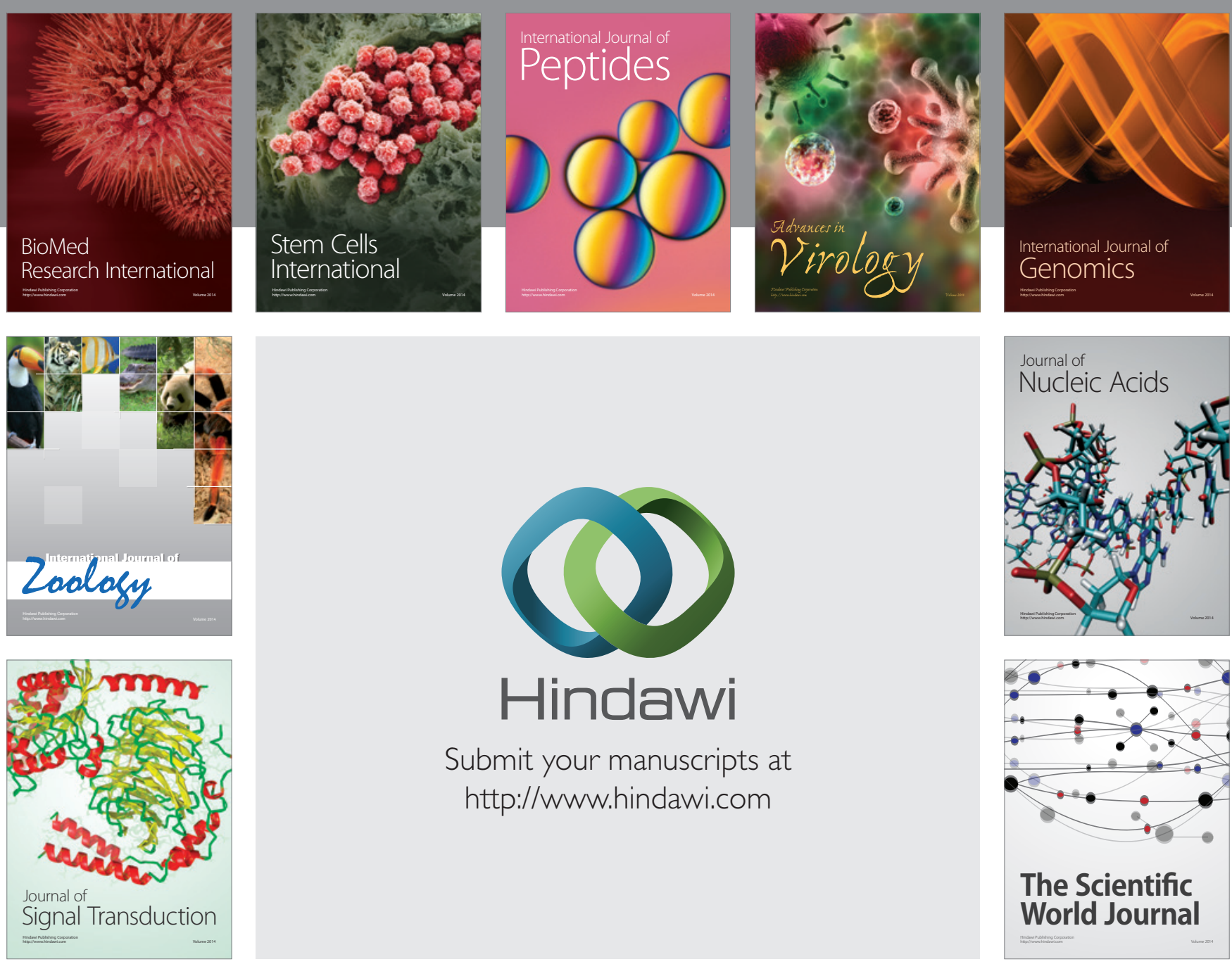

Submit your manuscripts at

http://www.hindawi.com
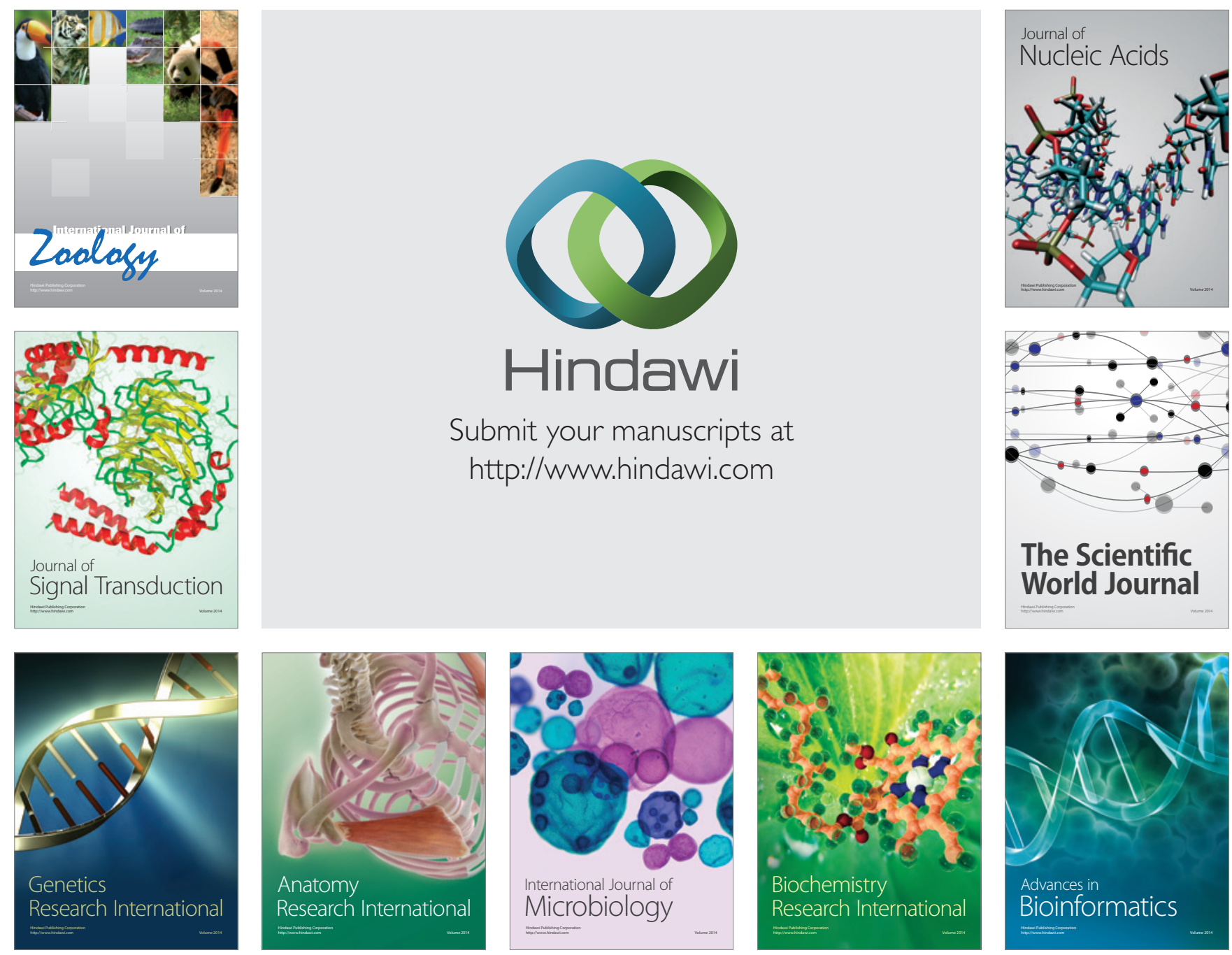

The Scientific World Journal
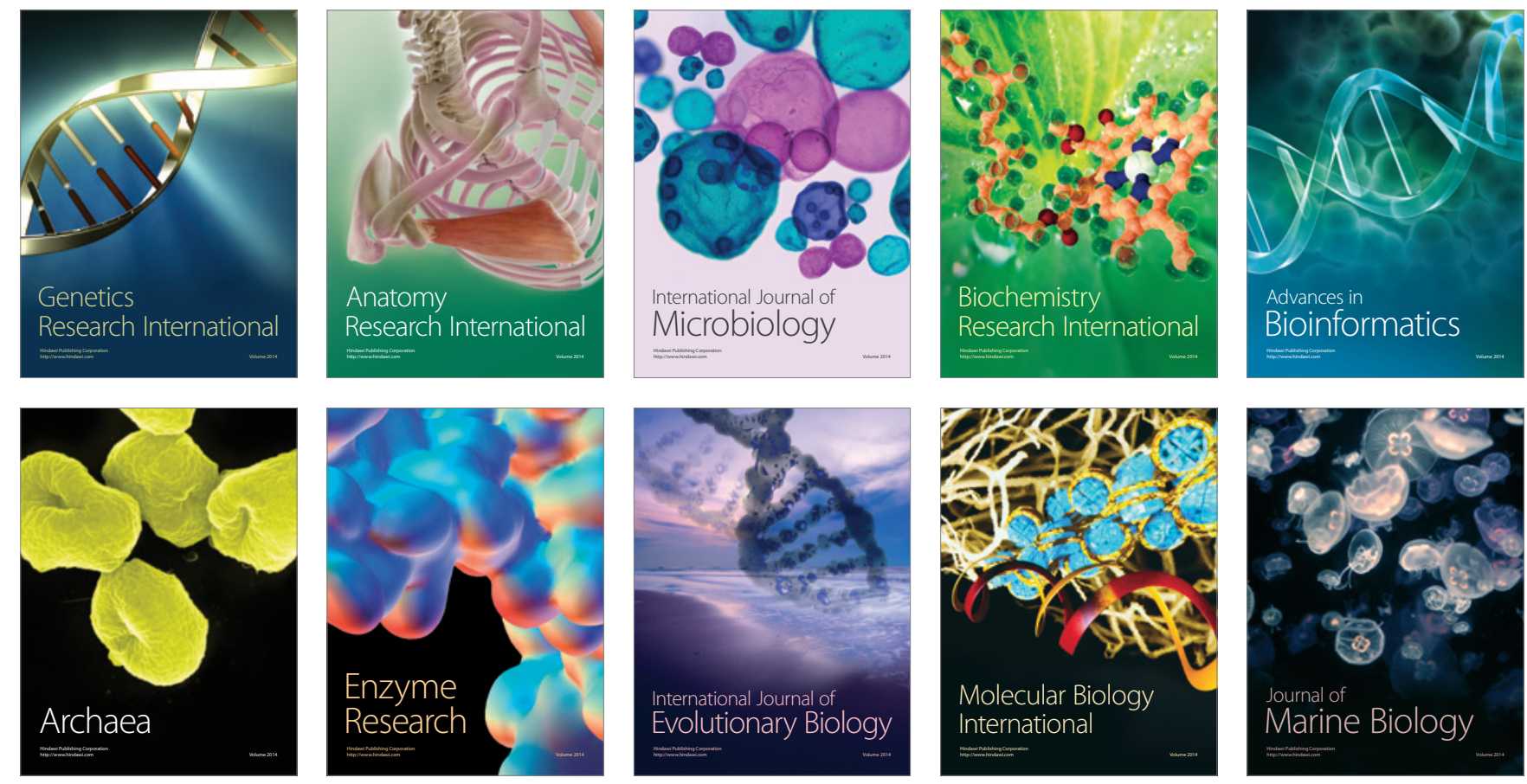